\title{
Photon antibunching in a cavity-QED system with two
}

\section{Rydberg-Rydberg interaction atoms*}

\author{
Tong Huang ${ }^{1}$, Lei $\operatorname{Tan}^{1,2} \dagger$ \\ ${ }^{1}$ Lanzhou Center for Theoretical Physics, Key Laboratory of Theoretical Physics \\ of Gansu Province,Lanzhou University, Lanzhou, Gansu 730000, China \\ ${ }^{2}$ Key Laboratory for Magnetism and Magnetic Materials of the Ministry of Education, \\ Lanzhou University, Lanzhou 730000, China
}

August 24, 2021

\begin{abstract}
We propose how to achieve strong photon antibunching effect in a cavity-QED system coupled with two Rydberg-Rydberg interaction atoms. Via calculating the equal time second order correlation function $g^{(2)}(0)$, we find that the unconventional photon blockade and the conventional photon blockade appear in the atom-driven scheme, and they are both significantly affected by the Rydberg-Rydberg interaction. We also find that under appropriate parameters, the photon antibunching and the mean photon number can be significantly enhanced by combining the conventional photon blockade and the unconventional photon blockade. In the cavity-driven scheme, the existence of the Rydberg-Rydberg interaction severely destroys the photon antibunching under the unconventional photon blockade mechanism. These results will help to guide the implementation of the single photon emitter in the Rydberg atoms-cavity system.
\end{abstract}

Keywords: photon antibunching, Rydberg-Rydberg interaction, unconventional photon blockade.

PACS: $03.67 . \mathrm{Hk}, 42.50 .-\mathrm{p}$.

\section{Introduction}

Photon antibunching is a quantum mechanic effect in which emitted photons tend to be detected one by one ${ }^{[1-5]}$. This important effect can be used to implement single photon emitter, which has important applications in quantum information processing ${ }^{[6,7]}$ and quantum computing ${ }^{[8,9]}$. There are two methods to achieve strong photon antibunching in the cavity-QED system. One is called the conventional photon blockade (PB): A strongly coupled system between an optical cavity and another nonlinear degree of freedom possesses an anharmonic ladder in the energy spectrum. Thus, it can produce the photon antibunching when the singlephoton nonlinearity is larger than the mode linewidth ${ }^{[10]}$. In the past few decades, a sequence of experimental

\footnotetext{
*Project supported by the National Natural Science Foundation of China (Grant No.11874190).

${ }^{\dagger}$ Corresponding author. E-mail:tanlei@lzu.edu.cn
} 
and theoretical works related to the PB have been investigated in various systems, such as cavity-atom systems ${ }^{[11,12]}$, superconducting qubit systems ${ }^{[13,14]}$, optomechanical systems ${ }^{[15-17]}$, etc.

In 2010, Liew and Savona found another mechanism called the unconventional photon blockade (UPB) originated from quantum interference which leads the probability amplitude of the two photon state to zero [18]. The UPB usually requires to add additional degrees of freedom, such as auxiliary cavities ${ }^{[19-21]}$ or two-level atoms (TLAs) ${ }^{[22,23]}$ into the system in order to establish multiple transition pathways to realize the destructive interference. In contrast with the $\mathrm{PB}$, this interference-based photon blockade mechanism can achieve strong photon antibunching only requiring weak nonlinearity ${ }^{[19,24-26]}$. Due to this significant feature, a large number of works related to the UPB have been reported extensively in various system, such as single mode cavity with second order nonlinearity [27], single mode cavity with Kerr-type nonlinearity [18,28,29], two-level emitter-cavity system [23], single mode cavity including a degenerate optical parametric amplifier [30], coupled optomechanical system ${ }^{[31,32]}$ and Gaussian squeezed states ${ }^{[33]}$.

Rydberg atoms with large principal quantum numbers $n$ have become an important tool of quantum information processing ${ }^{[34,35]}$. Rydberg blockade appears in the Rydberg atoms ensemble because of the strong Rydberg-Rydberg interaction, where Rydberg excitation of one atom prevents the excitation of atoms within the blockade radius. This phenomenon can be used to induce strong optical nonlinearity [36] which generates nonclassical states of light in the Rydberg atoms ensemble and the Rydberg atoms-cavity system [37-39]. In addition, photon antibunching induced by quantum interference can also be obtained in TLAs-cavity system ${ }^{[40,41]}$. Thus, one expects to obtain strong photon antibunching in two level Rydberg atoms-cavity system by combining the above two effects.

This paper is organized as follows. In Sec. II, we illustrate the theoretical model and Hamiltonian of the system, the cavity-atom interaction part of the Hamiltonian is rewritten in terms of the collective operators to obtain an intuitive picture of the photon transition pathways. In Sec. III, we calculate the equal time second order correlation function under the cavity-driven and the atom-driven scheme, respectively. Numerical and analytical methods are used to obtain the effect of Rydberg-Rydberg interaction on the photon antibunching. In addition, a summary is given in Sec. IV.

\section{Model}

As shown in Fig. 1, the system consists of a single mode cavity and two Rydberg coupling atoms trapped alone the axis of the cavity. A continuous wave coherent field is applied to drive the atoms (cavity). The total Hamiltonian of the system can be written in the rotating frame with the laser frequency $\omega_{L}$ as

$$
\hat{H}=-\sum_{j=1,2} \Delta_{a} \sigma_{r r}^{j}-\Delta_{c} a^{\dagger} a+\sum_{j=1,2} g_{j}\left(\sigma_{r g}^{j} a+\sigma_{g r}^{j} a^{\dagger}\right)+V \sigma_{r r}^{1} \sigma_{r r}^{2}+\hat{H}_{d}
$$

where $a\left(a^{\dagger}\right)$ is the annihilation (creation) operator of the cavity mode with resonant frequency $\omega_{c}$. $\sigma_{m n}^{j}=$ $|m\rangle_{j j}\langle n|$ with $m, n=r, g$ and the transition frequency between the ground state $|g\rangle$ and the Rydberg state $|r\rangle$ is denoted by $\omega_{a} \cdot g_{j}=g \cos \left(k x_{j}\right)$ is the position dependent coupling strength between the cavity field and the $j$ th atom at the point $x_{j}{ }^{[42]} . V$ represents the Rydberg coupling strength between two Rydberg atoms at a distance of $d$, it takes the form $V \propto \frac{1}{d^{6}}$ when Rydberg atoms interact through a van der Waals potential and the form $V \propto \frac{1}{d^{3}}$ when Rydberg atoms couple through the dipole-dipole interaction . The last term $\hat{H}_{d}$ in Eq. 


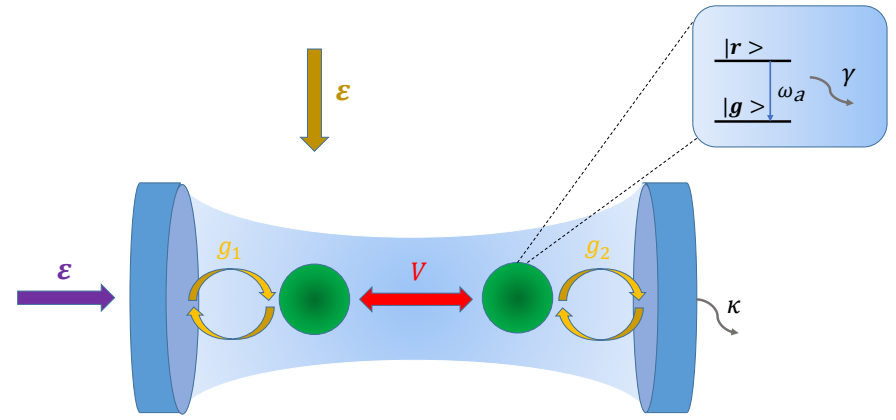

Figure 1: Scheme of a dissipative system: two identical two level Rydberg atoms are trapped in the single model cavity. The atoms are pumped in the direction perpendicular to the axis of the cavity and the cavity is pumped in the direction parallel to the axis of the cavity.

(1) describes cavity-driven term with $\varepsilon\left(a+a^{\dagger}\right)$ and atom-driven term with $\varepsilon \sum_{j=1,2}\left(\sigma_{r g}^{j}+\sigma_{g r}^{j}\right)$, respectively. Here $\Delta_{c}=\omega_{L}-\omega_{c}$ denotes the laser-cavity frequency detuning. $\Delta_{a}=\omega_{L}-\omega_{a}$ is the laser-atom frequency detuning and $\varepsilon$ represents the pump laser amplitude.

Defining the collective operators ${ }^{[43]}$ as

$$
D_{ \pm}^{\dagger}=\frac{1}{\sqrt{2}}\left(\sigma_{r g}^{1} \pm \sigma_{r g}^{2}\right)
$$

and the atom-cavity interaction part of the Hamiltonian in Eq. (1) can be reformulated as

$$
\begin{gathered}
\hat{H}_{I}=\hat{H}_{+}+\hat{H}_{-} . \\
\hat{H}_{ \pm}=\frac{g}{\sqrt{2}}\left(\cos \left(k x_{1}\right) \pm \cos \left(k x_{2}\right)\right)\left(a D_{ \pm}^{\dagger}+a^{\dagger} D_{ \pm}\right) .
\end{gathered}
$$

where $\hat{H}_{+}$represents the coupling of the symmetric state and the cavity mode leading to the transitions $\mid g g, n+$ $2\rangle \longleftrightarrow|+, n+1\rangle \longleftrightarrow|r r, n\rangle . \hat{H}_{-}$represents the coupling of the antisymmetric state and the cavity mode, which gives rise to the transitions $|g g, n+2\rangle \longleftrightarrow|-, n+1\rangle \longleftrightarrow|r r, n\rangle$. If we choose the distance between two Rydberg aroms as $d=x_{2}-x_{1}=n \lambda(n=0,1,2 \ldots), \hat{H}_{-}$vanishes and the cavity mode only couples via $|+\rangle$. Thus, the Hamiltonian of the system can be rewritten as

$$
\hat{H}_{r}=-\sum_{j=1,2} \Delta_{a} \sigma_{r r}^{j}-\Delta_{c} a^{\dagger} a+g \sum_{j=1,2}\left(\sigma_{r g}^{j} a+\sigma_{g r}^{j} a^{\dagger}\right)+V \sigma_{r r}^{1} \sigma_{r r}^{2}+\hat{H}_{d}
$$

In the following, we will discuss the photon antibunching of the system with atom-driven and cavity-driven scheme, respectively.

\section{Results and discussions}

\section{1. atom-driven scheme}

First, in order to get instructive understanding of the photon antibunching, one seeks to analytical description of the equal time second order correlation function $g^{(2)}(0)$, which measures the photon statistic distribution 


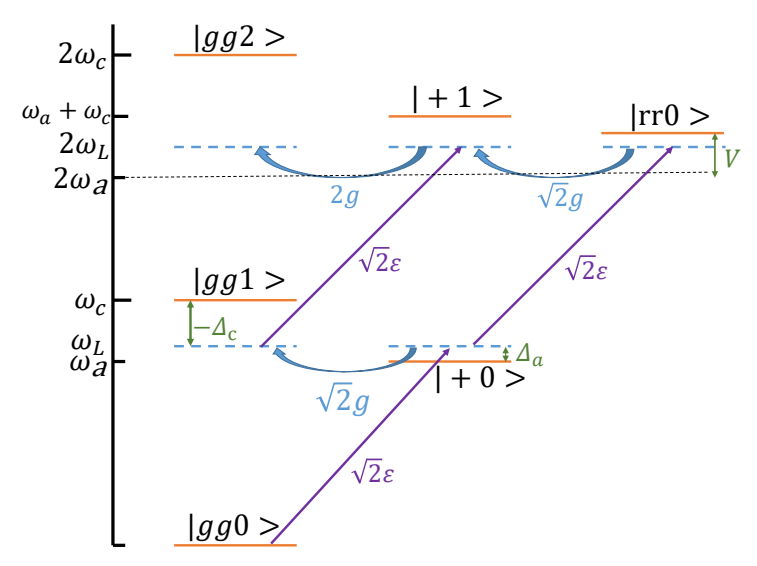

Figure 2: Transition pathways with atom-driven scheme

of the cavity mode. Within the weak driving condition, only lower photons excitation states are occupied, then one can express the wave function of the system with truncated state as follows

$$
\begin{array}{r}
\mid \psi(t\rangle)=C_{g g 0}(t)|g g 0\rangle+C_{g g 1}(t)|g g 1\rangle+C_{g g 2}(t)|g g 2\rangle+ \\
C_{+0}(t)|+0\rangle+C_{+1}(t)|+1\rangle+C_{r r 0}(t)|r r\rangle .
\end{array}
$$

the coefficient $C_{a a n}(t)$ represents the probability amplitude of the state $|a a n\rangle=|a a\rangle \otimes|n\rangle$, where $|a a\rangle(a a=$ $g g, r r,+)$ is the collective states of the two coupled Rydberg atoms and $|n\rangle(n=0,1,2)$ is the Fock basis for the cavity mode. The value $\left|C_{a a n}(t)\right|^{2}$ denotes the probability of the corresponding state. In order to obtain the value of the probability amplitude, we need to solve the time-dependent Schrödinger equation $i \hbar \frac{\partial}{\partial_{t}}|\psi(t)\rangle=\widetilde{H}|\psi(t)\rangle$, where $\widetilde{H}$ is the effective non-Hermitian Hamiltonian

$$
\widetilde{H}=\hat{H}_{r}-\frac{i \kappa a^{\dagger} a}{2}-\frac{i \gamma}{2}\left(\sigma_{1}^{\dagger} \sigma_{1}+\sigma_{2}^{\dagger} \sigma_{2}\right) .
$$

$\kappa$ and $\gamma$ are loss rates of the cavity and the atoms, respectively. Substituting Eq. (6) and Eq. (7) into the Schrödinger equation, one can obtain a set of equations of the time-dependent coefficients $(\hbar=1)$

$$
\begin{aligned}
& i \dot{C}_{g g 1}=\sqrt{2} g C_{+0}+\sqrt{2} \varepsilon C_{+1}-\Delta_{c} C_{g g 1}-\frac{i \kappa}{2} C_{g g 1}, \\
& i \dot{C}_{g g 2}=2 g C_{+1}-2 \Delta_{c} C_{g g 2}-i \kappa C_{g g 2}, \\
& i \dot{C}_{r r 0}=\left(-2 \Delta_{a}+V-i \gamma\right) C_{r r 0}+\sqrt{2} g C_{+1}+\sqrt{2} \varepsilon C_{+0}, \\
& i \dot{C}_{+0}=-\Delta_{a} C_{+0}+\sqrt{2} g C_{g g 1}+\sqrt{2} \varepsilon\left(C_{g g 0}+C_{r r 0}\right)-\frac{i \gamma}{2} C_{+0}, \\
& i \dot{C}_{+1}=-\left(\Delta_{a}+\Delta_{c}\right) C_{+1}+\sqrt{2} g C_{r r 0}+2 g C_{g g 2}+\sqrt{2} \varepsilon C_{g g 1}-\frac{i(\gamma+\kappa)}{2} C_{+1} .
\end{aligned}
$$

Under the weak driving condition, given that $\left\{C_{g g 0}\right\} \gg\left\{C_{g g 1}, C_{+0}\right\} \gg\left\{C_{g g 2}, C_{+1}, C_{r r 0}\right\}$ and set $C_{g g 0}=1$, the steady state solution can be obtained as follows

$$
\begin{aligned}
& C_{g g 1}=-\frac{8 g \varepsilon}{M}, \\
& C_{g g 2}=\frac{\left.16 \sqrt{2} g^{2} \varepsilon^{2}\left[\kappa+2 \gamma-4 i \Delta_{a}-2 i \Delta_{c}+2 i V\right)\right]}{M N} .
\end{aligned}
$$

where $M=8 g^{2}-2 i \kappa \Delta_{a}-2 i \gamma \Delta_{c}-4 \Delta_{a} \Delta_{c}+\kappa \gamma, N=4 g^{2} \kappa-16 i g^{2} \Delta_{a}+2 i \kappa^{2} \Delta_{a}-4 \kappa \Delta_{a}^{2}-8 i g^{2} \Delta_{c}+8 i \Delta_{a}^{2} \Delta_{c}+$ $8 i \Delta_{a} \Delta_{c}^{2}+8 g^{2} \gamma-\kappa^{2} \gamma-4 \Delta_{c}^{2} \gamma-\kappa \gamma^{2}+2 i \Delta_{c} \gamma^{2}+8 i g^{2} V-i \kappa^{2} V+2 \kappa \Delta_{a} V-4 i \Delta_{a} \Delta_{c} V-4 i \Delta_{c}^{2} V-i \kappa \gamma V+2 i \Delta_{c} \gamma V$. 
Under the condition of the large laser-atom (laser-cavity) frequency detuning limit: $\Delta_{a}, \Delta_{c} \gg \gamma, \kappa$, an analytical expression of the equal time second order correlation function can be represented as

$$
g^{(2)}(0)=\frac{<a^{\dagger} a^{\dagger} a a>}{<a^{\dagger} a>^{2}} \simeq \frac{2\left|C_{g g 2}\right|^{2}}{\left|C_{g g 1}\right|^{4}} \simeq-\frac{16\left(2 g^{2}-\Delta_{a} \Delta_{c}\right)^{2}\left(2 \Delta_{a}+\Delta_{c}-V\right)^{2}}{N^{2}} .
$$

which quantifies the joint probability of detecting two photons at the same time. In the limitation of $g^{2}(0) \rightarrow 0$, one can obtain the optimal conditions of the photon antibunching

$$
\begin{aligned}
\Delta_{a} & =\frac{1}{2}\left(V-\Delta_{c}\right), \\
\Delta_{a} & =\frac{2 g^{2}}{\Delta_{c}} .
\end{aligned}
$$

The first expression in Eq. (11) is an optimal condition for the UPB induced by quantum interference, which can be obtained when the probability amplitude $C_{g g 2}=0$. One can see that it's relevant to the laser-atom frequency detuning, laser-cavity frequency detuning, and Rydberg coupling strength. As shown in Fig. 2, two transition pathways $|g g 0\rangle \stackrel{\sqrt{2} \xi}{\longrightarrow}|+0\rangle \stackrel{\sqrt{2} g}{\longrightarrow}|g g 1\rangle \stackrel{\sqrt{2} \xi}{\longrightarrow}|+1\rangle \stackrel{2 g}{\longrightarrow}|g g 2\rangle$ and $|g g 0\rangle \stackrel{\sqrt{2} \xi}{\longrightarrow}|+0\rangle \stackrel{\sqrt{2} \xi}{\longrightarrow}|r r 0\rangle \stackrel{\sqrt{2} g}{\longrightarrow}|+1\rangle \stackrel{2 g}{\longrightarrow}$ $|g g 2\rangle$ are distinguishable. Thus, destructive interference arises between the two pathways and one can expect to get a strong UPB effect ${ }^{[42]}$. The second formula in Eq. (11) is the optimal condition of the PB. As shown in previous studies ${ }^{[40]}$, one can obtain a PB effect when the cavity-atom coupling strength $g$ is large enough.

Next, we will numerically calculate the equal time second order correlation function $g^{(2)}(0)$ by solving the master equation of the system

$$
i \frac{\partial \hat{\rho}}{\partial t}=[\hat{H}, \hat{\rho}]-i \sum_{j=1,2} \frac{\gamma_{j}}{2} \mathcal{D}\left[\sigma_{j}\right] \hat{\rho}-i \frac{\kappa}{2} \mathcal{D}[a] \hat{\rho} .
$$

where $\mathcal{D}[q] \hat{\rho}=2 q \rho q^{\dagger}-q^{\dagger} q \hat{\rho}-\hat{\rho} q^{\dagger} q$ denotes the Liouvillian superoperator that represents the loss of the Rydberg atoms (cavity), the collective damping of the Rydberg atoms is not considered for simplicity. The steady state solution of the equal time second order correlation function $g^{(2)}(0)$ is expressed as

$$
g^{(2)}(0)=\frac{\operatorname{Tr}\left[\hat{\rho} a^{\dagger} a^{\dagger} a a\right]}{\left(\operatorname{Tr}\left[\hat{\rho} a^{\dagger} a\right]\right)^{2}} .
$$

In the experiment, we consider two ${ }^{87} R b$ atoms placed in a plane cavity to produce nonclassical light. Such a single photon emitter can be realized by implementing a two-dimensional optical lattice in a cavity ${ }^{[44]}$, and the two-dimensional optical lattice consists of a red-detuned laser beam perpendicular to the cavity axis and a blue-detuned laser beam which alone the cavity axis. Rydberg atoms are distributed at several lattice sites, so they have well-defined positions. Moreover, excess Rydberg atoms in the optical lattice can be removed with a resonant push-out beam. The experimentally achievable parameters of the distance between two ${ }^{87} R b$ atoms can be adjusted from $15 \mu \mathrm{m}$ to $4 \mu \mathrm{m}{ }^{[45]}$. For the Rydberg states $|r\rangle=\left|62 D_{\frac{3}{2}}\right\rangle$, the van der Waals interaction cofficient is $C_{6}=730 G H z \cdot \mu m^{6}$, and the Rydberg coupling strength $V$ can be tuned continuously between $2 \pi \times(0.01,28.33) M H z{ }^{[45]}$. In addition, for principal quantum number $n \sim 60$, the ${ }^{87} R b$ atoms $\left|n D_{\frac{3}{2}}\right\rangle$ state has a lifetime of $203.80 \mu \mathrm{s}$ (the spontaneous decay rate is $\gamma=2 \pi \times 0.4 K H z^{[46,47]}$ ). The pump laser amplitude $\varepsilon$ can be adjusted from $2 \pi \times 500 \mathrm{KHz}$ to $2 \pi \times 5 \mathrm{MHz}$. Moreover, the cavity QED experiments with ${ }^{87} \mathrm{Rb}$ atoms provide us other parameters: $(g, \kappa)=2 \pi \times(7.8,2.5) M H z{ }^{[44]}$. Next, we numerically calculate the photon antibunching by using the above experimental parameters. 


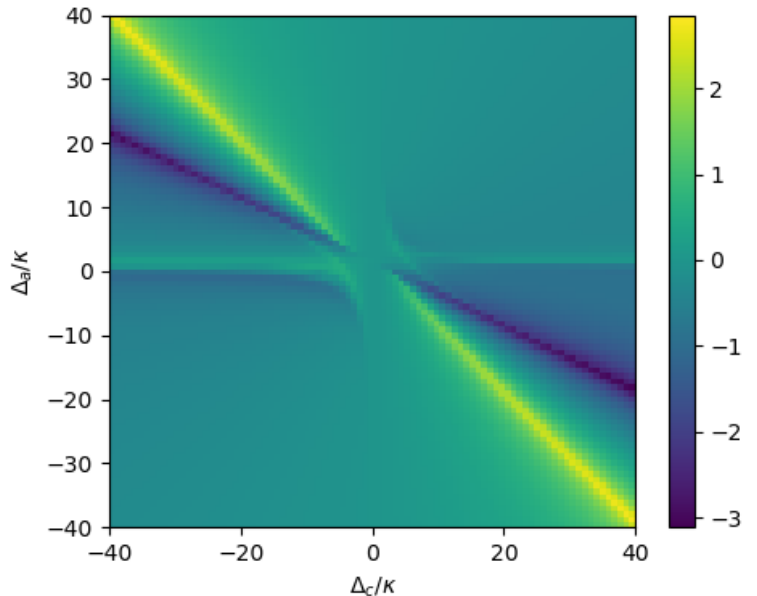

(a)

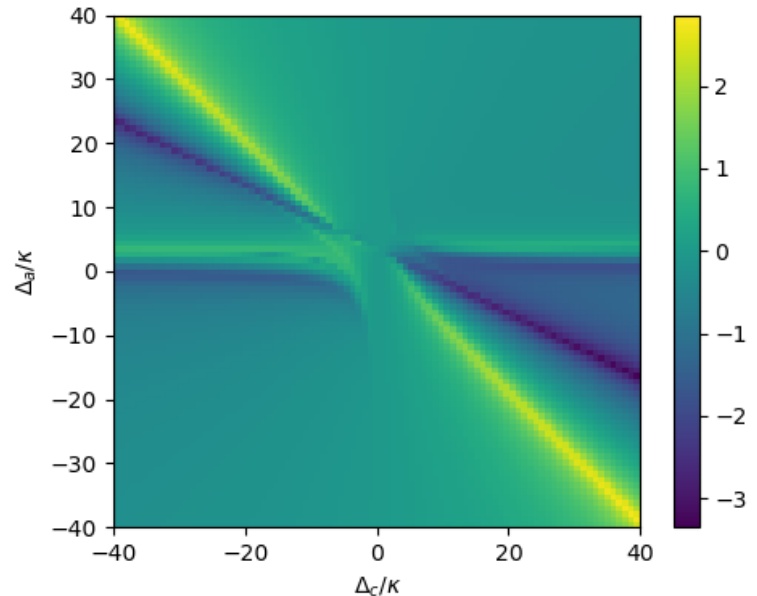

(b)

Figure 3: (a) Plot of $\log _{10}\left[g^{(2)}(0)\right]$ as a function of $\Delta_{c} / \kappa$ and $\Delta_{a} / \kappa$ with $V=2 \kappa$. (b) Plot of $\log _{10}\left[g^{(2)}(0)\right]$ as a function of $\Delta_{c} / \kappa$ and $\Delta_{a} / \kappa$ with $V=6 \kappa$. Other parameters take as $\gamma=2 \pi \times 0.4 K H z, \kappa=2 \pi \times 2.5 M H z$, $\varepsilon=0.4 \kappa$ and $g=2 \pi \times 7.8 M H z$.

In order to show the dependence of the $g^{(2)}(0)$ on the Rydberg coupling strength $V$, one plots the equal time second order correlation function $g^{(2)}(0)$ (in logarithmic units) as a function of $\Delta_{a} / \kappa$ and $\Delta_{c} / \kappa$ with the Rydberg coupling strength $V=2 \kappa$ and $6 \kappa$. The atom-driven strength $\varepsilon$ is assigned to $0.4 \kappa$ for weak driving condition. From Figs. 3(a) and 3(b), one can see that the UPB effect appears and the position of the UPB can be changed by the Rydberg coupling strength $V$. We also find that the PB effect becomes significant in the case of the large cavity-atom coupling strength $g$. These numerical results are consistent with the analytical solution for the optimal conditions in Eq. (11).

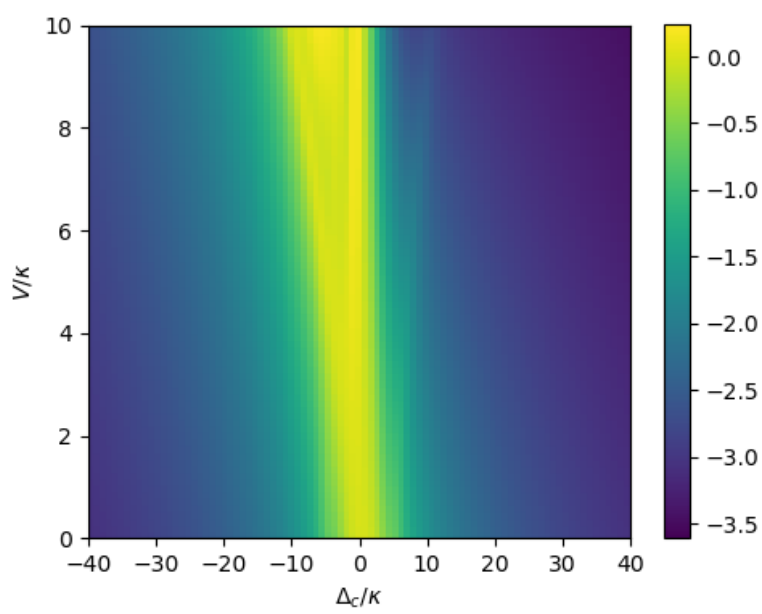

(a)

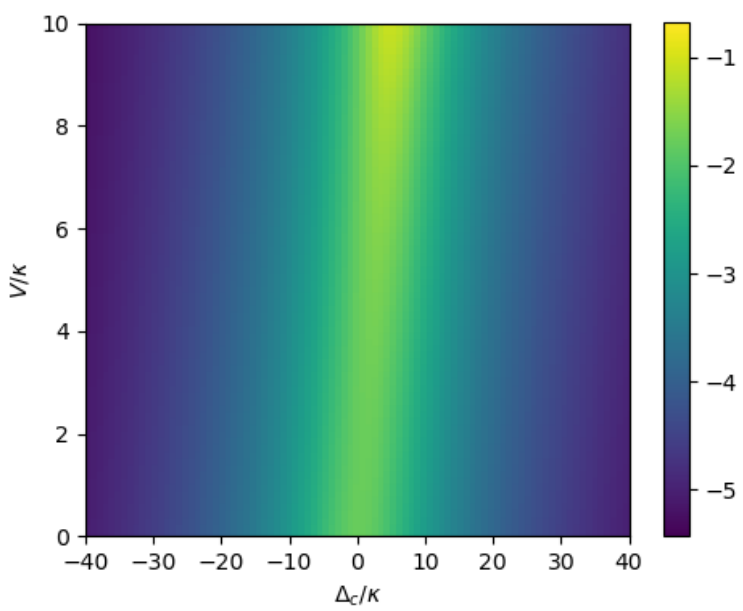

(b)

Figure 4: (a) Plot of $\log _{10}\left[g^{(2)}(0)\right]$ as a function of $\Delta_{c} / \kappa$ and $V / \kappa$ with $\Delta_{a}=-\frac{1}{2}\left(\Delta_{c}-V\right)$. (b) Plot of the mean photon number $\left\langle a^{\dagger} a\right\rangle$ (log scale) as a function of $\Delta_{c} / \kappa$ and $V / \kappa$ with $\Delta_{a}=-\frac{1}{2}\left(\Delta_{c}-V\right)$. Other parameters take as $\gamma=2 \pi \times 0.4 \mathrm{KHz}, \kappa=2 \pi \times 2.5 \mathrm{MHz}, \varepsilon=0.4 \kappa$ and $g=2 \pi \times 7.8 \mathrm{MHz}$. 
To further clarify the effect of the Rydberg coupling strength $V$ on the UPB, we plot the equal time second order correlation function $g^{(2)}(0)$ and the mean photon number $\left\langle a^{\dagger} a\right\rangle$ as a function of $\Delta_{c} / \kappa$ and $V / \kappa$ with $\Delta_{a}=-\frac{1}{2}\left(\Delta_{c}-V\right)$ in Figs. 4(a) and 4(b). From Fig. 4(a), one can see that photon antibunching based on the quantum interference is greatly enhanced by the laser-cavity frequency detuning $\Delta_{c}$. Under the condition of the large negative laser-cavity frequency detuning limit, the smaller the Rydberg coupling strength $V$ the stronger the UPB effect is. In the limit of the large positive laser-cavity frequency detuning, there is also a strong UPB effect at any value of $V$. Contrary to the second-order correlation function $g^{(2)}(0)$, the mean photon number $\left\langle a^{\dagger} a\right\rangle$ in the cavity is suppressed by the laser-cavity frequency detuning. This brings difficulties to the detection of single photons under strong photon antibunching conditions caused by quantum interference.

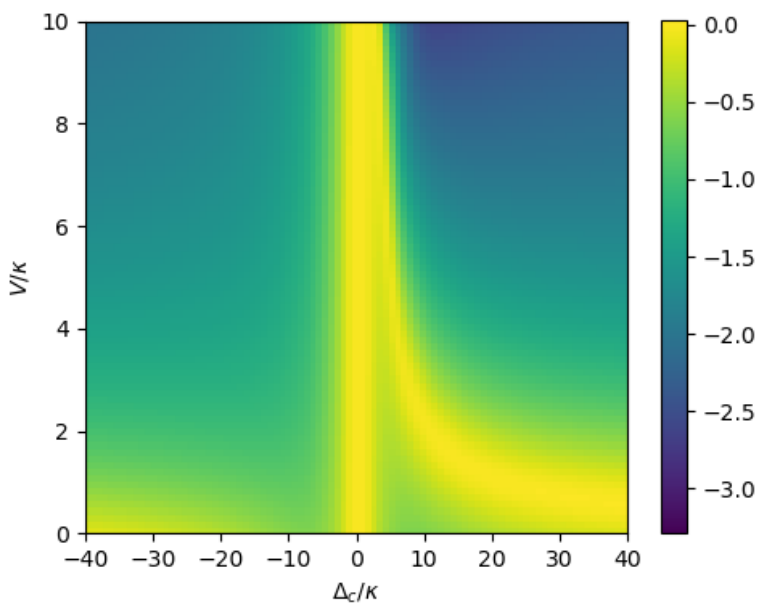

(a)

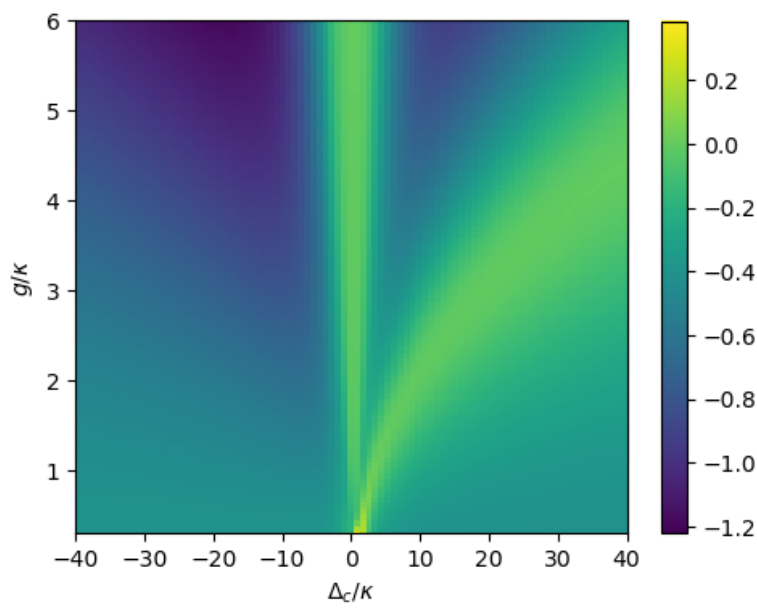

(b)

Figure 5: (a) Plot of $\log _{10}\left[g^{(2)}(0)\right]$ as a function of $\Delta_{c} / \kappa$ and $V / \kappa$ with $\Delta_{a}=\frac{2 g^{2}}{\Delta_{c}}$, the atom-cavity coupling strength is given by $g=2 \pi \times 7.8 \mathrm{MHz}$. (b) Plot of $\log _{10}\left[g^{(2)}(0)\right]$ as a function of $\Delta_{c} / \kappa$ and $g / \kappa$ with $\Delta_{a}=\frac{2 g^{2}}{\Delta_{c}}$, the Rydberg coupling strength is given by $V=\kappa$. Other parameters take as $\gamma=2 \pi \times 0.4 K H z, \kappa=2 \pi \times 2.5 M H z$, $\varepsilon=0.4 \kappa$.

Next, we investigate the effect of the Rydberg coupling strength $V$ and the cavity-atom coupling strength $g$ on the PB under the optimal condition $\Delta_{a}=\frac{2 g^{2}}{\Delta_{c}}$. In Fig. $5(\mathrm{a})$, we plot the equal time second order correlation function $g^{(2)}(0)$ as a function of $\Delta_{c} / \kappa$ and $V / \kappa$. It's clear that strong photon antibunching based on the energylevel anharmonicity can be obtained in the positive laser-cavity frequency detuning interval when the Rydberg coupling strength $V$ is large enough. Fig. 5(b) displays the equal time second order correlation function $g^{(2)}(0)$ as a function of $\Delta_{c} / \kappa$ and $g / \kappa$. We find that under the PB optimal condition, the PB effect can be enhanced with the increase of coupling strength $g$ at the large laser-cavity frequency detuning limit.

The above analysis in Fig. 4 and Fig. 5 are based on the separate consideration of the UPB and the PB effect. Thus, we can only get a small mean photon number $\left\langle a^{\dagger} a\right\rangle$ when the equal time second order correlation function $g^{(2)}(0)$ is extremely small. It's an adverse result for single photons detection. Here, we consider whether we can obtain stronger photon antibunching and larger mean photon number in the cavity by combining the UPB effect and the PB effect. In Figs. 6(a) and 6(b), we plot the equal time second order correlation function $g^{(2)}(0)$ and the mean photon number $\left\langle a^{\dagger} a\right\rangle$ as a function of $\Delta_{c} / \kappa$ and $V / \kappa$ with $\Delta_{a}=-\frac{1}{2}\left(\Delta_{c}-V\right)$. As shown in 


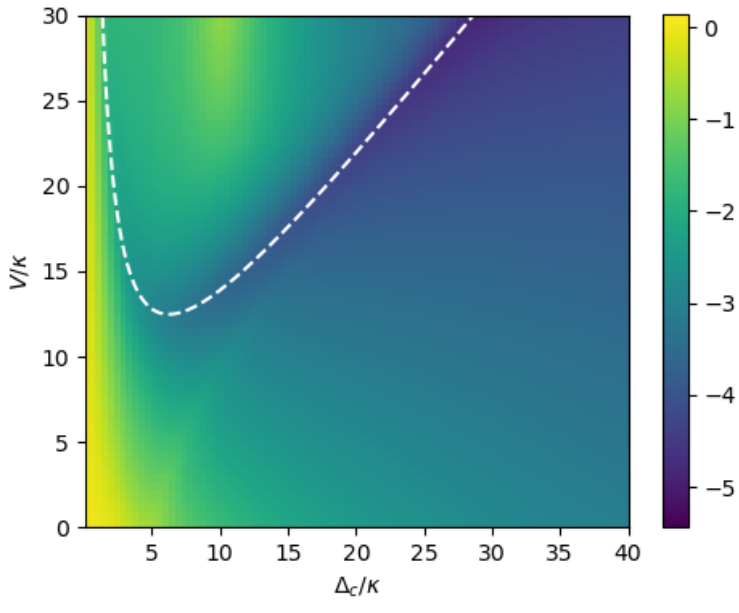

(a)

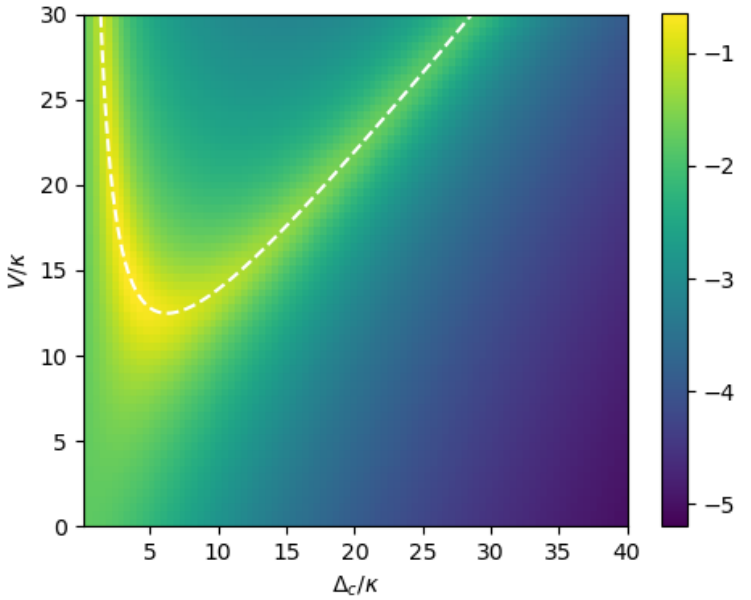

(b)

Figure 6: (a) Plot of $\log _{10}\left[g^{(2)}(0)\right]$ as a function of $\Delta_{c} / \kappa$ and $V / \kappa$ with $\Delta_{a}=-\frac{1}{2}\left(\Delta_{c}-V\right)$. (b) Plot of $\left\langle a^{\dagger} a\right\rangle$ (log scale) as a function of $\Delta_{c} / \kappa$ and $V / \kappa$ with $\Delta_{a}=-\frac{1}{2}\left(\Delta_{c}-V\right)$. Other parameters take as $\gamma=2 \pi \times 0.4 K H z$, $\kappa=2 \pi \times 2.5 \mathrm{MHz}, \varepsilon=0.4 \kappa$ and $g=2 \pi \times 7.8 \mathrm{MHz}$. The white dashed line is depicted by the function $V=\Delta_{c}+\frac{4 g^{2}}{\Delta_{c}}$.

Fig. 6(a), the value of the equal time second order correlation function $g^{(2)}(0)$ at the minimum is about $10^{-5.5}$. As assumed above, a stronger photon antibunching based on the UPB and the PB mechanism appears when the parameters satisfy $V>4 g$. The white dashed line $\left(V=\Delta_{c}+\frac{4 g^{2}}{\Delta_{c}}\right)$ in Fig. 6(a) corresponds to the intersection of the UPB optimal condition and the PB optimal condition. It analytically marks the minimum value of the equal time second order correlation function $g^{(2)}(0)$ in the parameter space. In Fig. 6(b), we can see that under the condition that the parameters satisfy the UPB optimal condition, the mean photon number $\left\langle a^{\dagger} a\right\rangle$ in the cavity can reach the maximum simultaneously when the equal time second order correlation function $g^{(2)}(0)$ takes a minimum value.

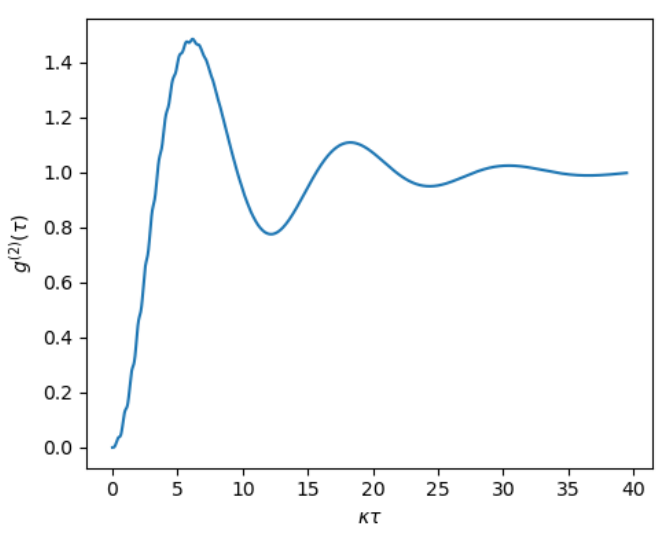

(a)

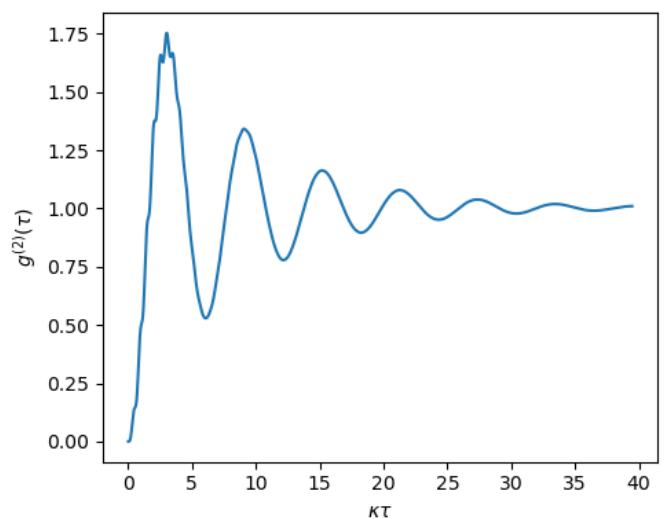

(b)

Figure 7: Plot of $g^{(2)}(\tau)$ as a function of $\kappa \tau$ under the optimal conditions in Eq. $(11)\left(\Delta_{c}=10 \kappa, \Delta_{a}=1.95 \kappa\right.$, $V=13.9 \kappa, g=2 \pi \times 7.8 \mathrm{MHz}$ ) for different pump laser amplitude. (a) $\varepsilon=0.2 \kappa$, (b) $\varepsilon=0.4 \kappa$. Other parameters are set as $\gamma=2 \pi \times 0.4 \mathrm{KHz}, \kappa=2 \pi \times 2.5 \mathrm{MHz}$. 
Finally, in order to fully characterize the quantum signatures of the single photon emitter, we need to consider the delayed second order correlation function in the steady state

$$
g^{(2)}(\tau)=\frac{\operatorname{Tr}\left[\hat{\rho} a^{\dagger}(0) a^{\dagger}(\tau) a(\tau) a(0)\right]}{\left(\operatorname{Tr}\left[\hat{\rho} a^{\dagger}(0) a(0)\right]\right)^{2}} .
$$

In Figs. $7(\mathrm{a})$ and $7(\mathrm{~b})$, we plot the delayed second order correlation function $g^{(2)}(\tau)$ for the UPB and the PB optimal conditions. One can observe that at $\tau=0, g^{(2)}(0)=0$, and the delayed second order correlation function $g^{(2)}(\tau)>g^{(2)}(0)$, i.e., fewer photon pairs are detected close together than further apart, it's a signature of the photon antibunching. We also find that $g^{(2)}(\tau)$ presents an oscillation behavior and the oscillation period is modulated by the pump laser amplitude $\varepsilon$. In addition, the value of $g^{(2)}(\tau)$ approaches unity for long time delay.

\section{2. cavity-driven scheme}

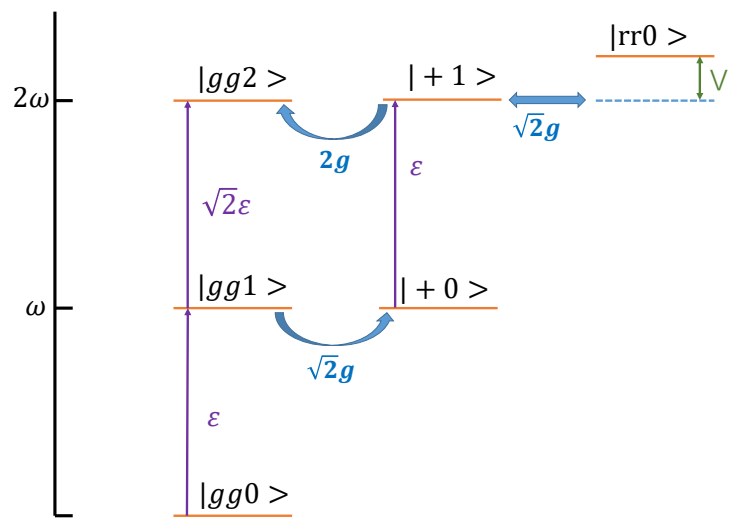

Figure 8: Transition pathways with cavity-driven scheme

Here, we consider the cavity-driven scheme with $\Delta_{a}=\Delta_{c}=0$, i.e., detunings of the two level Rydberg atoms transition frequency and the cavity resonant frequency from the driving laser vanish. Analytic expression for the time dependent coefficient can be obtained by solving the Schrödinger equations:

$$
\begin{aligned}
& i \dot{C}_{g g 1}=-\frac{i \kappa}{2} C_{g g 1}+\sqrt{2} g C_{+0}+\varepsilon C_{g g 0}+\sqrt{2} \varepsilon C_{g g 2}, \\
& i \dot{C}_{g g 2}=-i \kappa C_{g g 2}+2 g C_{+1}+\sqrt{2} \varepsilon C_{g g 1}, \\
& i \dot{C}_{r r 0}=(-i \gamma+V) C_{r r 0}+\sqrt{2} g C_{+1}, \\
& i \dot{C}_{+0}=-\frac{i \gamma}{2} C_{+0}+\sqrt{2} g C_{g g 1}+\varepsilon C_{+1}, \\
& i \dot{C}_{+1}=-\frac{i(\gamma+\kappa)}{2} C_{+1}+\sqrt{2} g C_{r r 0}+2 g C_{g g 2}+\varepsilon C_{+0} .
\end{aligned}
$$

The steady state solutions of Eq. (15) can be obtained as follows

$$
C_{g g 2}=\frac{2 \sqrt{2} \varepsilon^{2}\left[\left(4 \varepsilon^{2}+\gamma(\gamma+\kappa)\right)(\gamma+i V)-4 g^{2}(\gamma+2 i V)\right]}{N} .
$$


where $N=\left(4 \varepsilon^{2}+\kappa^{2}\right)\left(4 \varepsilon^{2}+\gamma(\gamma+\kappa)\right)(\gamma+i V)+32 g^{4}(\kappa+2 \gamma+2 i V)+4 g^{2}\left(\gamma \kappa(3 \kappa+4 \gamma)-4 \varepsilon^{2}(3 \gamma+4 i V)+2 i \kappa(\kappa+2 \gamma) V\right)$. Within the same methods mentioned above, one gets the equations

$$
\begin{aligned}
& 4 \varepsilon^{2}-4 g^{2}+\kappa \gamma+\gamma^{2}=0, \\
& V=0 .
\end{aligned}
$$

Here, Eq. (17) is the optimal condition of the UPB induced by the destructive interference, which means that in order to get a strong photon antibunching effect, the strength of the Rydberg-Rydberg interaction must always be zero. That is, a finite Rydberg coupling strength $V$ could weaken the photon antibunching.

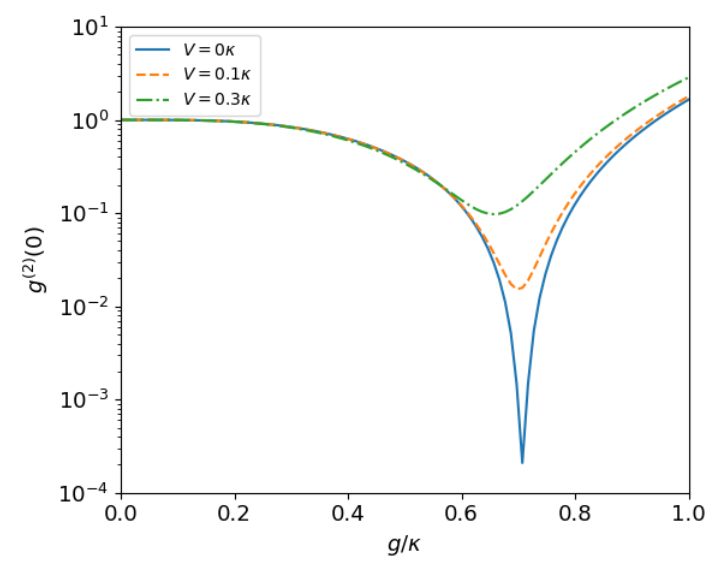

(a)

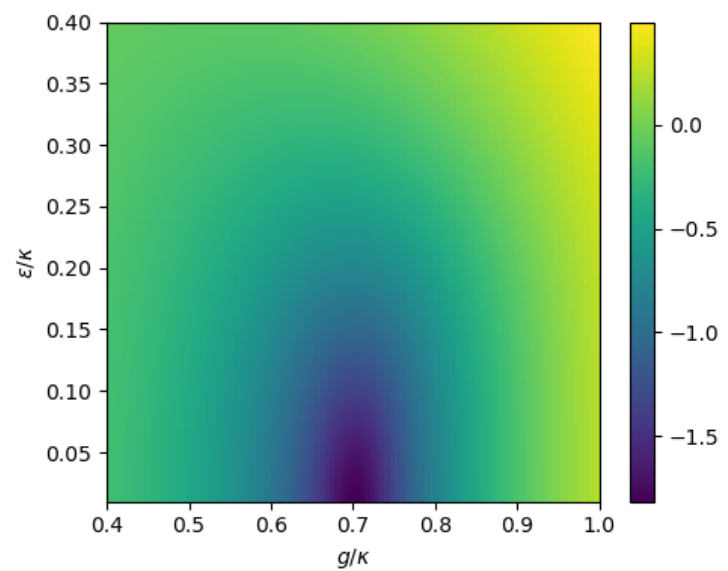

(b)

Figure 9: (a) Plot of $g^{(2)}(0)$ as a function of $g / \kappa$ with $V=0, V=0.1 \kappa$ and $V=0.3 \kappa$, respectively. Other parameters are given by $\varepsilon=0.01 \kappa$ and $\gamma=\kappa$. (b) Plot of $\log _{10}\left[g^{(2)}(0)\right]$ as a function of $g / \kappa$ and $\varepsilon / \kappa$, Other parameters are given by $V=0.1 \kappa$ and $\gamma=\kappa$.

In what follows, we will numerically study the UPB and compare it with the analytic results, which are shown in Fig. 9(a). Where the equal time second order correlation function $g^{(2)}(0)$ as a function of the cavityatom coupling strength $g$ is plotted for different Rydberg coupling strength. In Fig. 8, two excitation pathways are $|g g 0\rangle \stackrel{\varepsilon}{\longrightarrow}|g g 1\rangle \stackrel{\sqrt{2} \varepsilon}{\longrightarrow}|g g 2\rangle$ and $|g g 0\rangle \stackrel{\varepsilon}{\longrightarrow}|g g 1\rangle \stackrel{\sqrt{2} g}{\longleftrightarrow}|+0\rangle \stackrel{\varepsilon}{\longrightarrow}|+1\rangle(\stackrel{\sqrt{2} g}{\longleftrightarrow}|r r 0\rangle \stackrel{\sqrt{2} g}{\longleftrightarrow}|+1\rangle) \stackrel{2 g}{\longleftrightarrow}|g g 2\rangle$. The destructive interference results to a strong photon antibunching when the cavity-atom coupling strength $g \simeq 0.7 \kappa$ and $V=0$. When $V$ has a deviation from the optimal condition, the strength of the photon antibunching decreases rapidly as shown in Fig. 9(a). We also show the influence of cavity-driven strength on the UPB in Fig. 9(b). Where the equal time second order correlation function $g^{(2)}(0)$ as a function of the cavity-driven strength $\varepsilon / \kappa$ and the cavity-atom coupling strength $g / \kappa$. It can be seen that one can obtain strong photon antibunching effect with a weak cavity-driven strength. However, This would also lead to a small value of the mean photon number in the cavity, and hence of single photons number emitted from the cavity.

\section{Conclusion}

In summary, we have investigated the photon antibunching in a Rydberg atoms-cavity system under the atom-driven and cavity-driven scheme, respectively. By solving the Schrödinger equation and the Lindblad 
master equation, it can be found that in the case of the atom-driven, one can obtain a strong UPB effect when the laser-cavity frequency detuning is large enough. In the negative laser-cavity frequency detuning interval, the increase of Rydberg coupling strength weakens the UPB effect. In addition, Rydberg-Rydberg interaction can also result in strong PB effect in the positive laser-cavity frequency detuning region. It's worth noting that within the range of the appropriate parameters, while we get the extremely strong photon antibunching effect, the mean photon number in the cavity has also improved significantly. In the cavity-driven case, the increasing interatomic Rydberg-Rydberg interaction strength could weaken the photon antibunching rapidly. Our study may provide guidance for the experimental construction of single-photon sources in the Rydberg atoms-cavity system.

\section{References}

[1] H. Paul. Photon antibunching. Rev. Mod. Phys., 54:1061-1102, Oct 1982.

[2] H. J. Kimble, M. Dagenais, and L. Mandel. Photon antibunching in resonance fluorescence. Phys. Rev. Lett., 39:691-695, Sep 1977.

[3] X. T. Zou and L. Mandel. Photon-antibunching and sub-poissonian photon statistics. Phys. Rev. A, 41:475-476, Jan 1990.

[4] D. Stoler and B. Yurke. Generating antibunched light from the output of a nondegenerate frequency converter. Phys. Rev. A, 34:3143-3147, Oct 1986.

[5] M. H. Mahran and M. Venkata Satyanarayana. Bunching and antibunching properties of various coherent states of the radiation field. Phys. Rev. A, 34:640-643, Jul 1986.

[6] A. Kiraz, M. Atatüre, and A. Imamoğlu. Quantum-dot single-photon sources: Prospects for applications in linear optics quantum-information processing. Phys. Rev. A, 69:032305, Mar 2004.

[7] Pieter Kok, W. J. Munro, Kae Nemoto, T. C. Ralph, Jonathan P. Dowling, and G. J. Milburn. Linear optical quantum computing with photonic qubits. Rev. Mod. Phys., 79:135-174, Jan 2007.

[8] Brahim Lounis and Michel Orrit. Single-photon sources. Reports on Progress in Physics, 68(5):1129-1179, apr 2005.

[9] P. Yao, V.S.C. Manga Rao, and S. Hughes. On-chip single photon sources using planar photonic crystals and single quantum dots. Laser \& Photonics Reviews, 4(4):499-516, 2010.

[10] W. Leoński and R. Tanaś. Possibility of producing the one-photon state in a kicked cavity with a nonlinear kerr medium. Phys. Rev. A, 49:R20-R23, Jan 1994.

[11] Axel Kuhn, Markus Hennrich, and Gerhard Rempe. Deterministic single-photon source for distributed quantum networking. Phys. Rev. Lett., 89:067901, Jul 2002.

[12] Alexandre Le Boité, Myung-Joong Hwang, Hyunchul Nha, and Martin B. Plenio. Fate of photon blockade in the deep strong-coupling regime. Phys. Rev. A, 94:033827, Sep 2016. 
[13] Yu-xi Liu, Xun-Wei Xu, Adam Miranowicz, and Franco Nori. From blockade to transparency: Controllable photon transmission through a circuit-qed system. Phys. Rev. A, 89:043818, Apr 2014.

[14] A. J. Hoffman, S. J. Srinivasan, S. Schmidt, L. Spietz, J. Aumentado, H. E. Türeci, and A. A. Houck. Dispersive photon blockade in a superconducting circuit. Phys. Rev. Lett., 107:053602, Jul 2011.

[15] Xun-Wei Xu, Yuan-Jie Li, and Yu-xi Liu. Photon-induced tunneling in optomechanical systems. Phys. Rev. A, 87:025803, Feb 2013.

[16] Jie-Qiao Liao and Franco Nori. Photon blockade in quadratically coupled optomechanical systems. Phys. Rev. A, 88:023853, Aug 2013.

[17] P. Rabl. Photon blockade effect in optomechanical systems. Phys. Rev. Lett., 107:063601, Aug 2011.

[18] T. C. H. Liew and V. Savona. Single photons from coupled quantum modes. Phys. Rev. Lett., 104:183601, May 2010.

[19] H. Flayac and V. Savona. Unconventional photon blockade. Phys. Rev. A, 96:053810, Nov 2017.

[20] Y. H. Zhou, H. Z. Shen, and X. X. Yi. Unconventional photon blockade with second-order nonlinearity. Phys. Rev. A, 92:023838, Aug 2015.

[21] Motoaki Bamba, Atac Imamoğlu, Iacopo Carusotto, and Cristiano Ciuti. Origin of strong photon antibunching in weakly nonlinear photonic molecules. Phys. Rev. A, 83:021802, Feb 2011.

[22] Ming-Cui Li and Ai-Xi Chen. A photon blockade in a coupled cavity system mediated by an atom. Applied Sciences, 9(5), 2019.

[23] Xinyun Liang, Zhenglu Duan, Qin Guo, Cunjin Liu, Shengguo Guan, and Yi Ren. Antibunching effect of photons in a two-level emitter-cavity system. Phys. Rev. A, 100:063834, Dec 2019.

[24] H. J. Snijders, J. A. Frey, J. Norman, H. Flayac, V. Savona, A. C. Gossard, J. E. Bowers, M. P. van Exter, D. Bouwmeester, and W. Löffler. Observation of the unconventional photon blockade. Phys. Rev. Lett., 121:043601, Jul 2018.

[25] Cyril Vaneph, Alexis Morvan, Gianluca Aiello, Mathieu Féchant, Marco Aprili, Julien Gabelli, and Jérôme Estève. Observation of the unconventional photon blockade in the microwave domain. Phys. Rev. Lett., 121:043602, Jul 2018.

[26] G. T. Foster, S. L. Mielke, and L. A. Orozco. Intensity correlations in cavity qed. Phys. Rev. A, 61:053821, Apr 2000.

[27] Dario Gerace and Vincenzo Savona. Unconventional photon blockade in doubly resonant microcavities with second-order nonlinearity. Phys. Rev. A, 89:031803, Mar 2014.

[28] S Ferretti, V Savona, and D Gerace. Optimal antibunching in passive photonic devices based on coupled nonlinear resonators. New Journal of Physics, 15(2):025012, feb 2013. 
[29] Xun-Wei Xu and Yong Li. Tunable photon statistics in weakly nonlinear photonic molecules. Phys. Rev. A, 90:043822, Oct 2014.

[30] Bijita Sarma and Amarendra K. Sarma. Quantum-interference-assisted photon blockade in a cavity via parametric interactions. Phys. Rev. A, 96:053827, Nov 2017.

[31] Hui Wang, Xiu Gu, Yu-xi Liu, Adam Miranowicz, and Franco Nori. Tunable photon blockade in a hybrid system consisting of an optomechanical device coupled to a two-level system. Phys. Rev. A, 92:033806, Sep 2015.

[32] Dong-Yang Wang, Cheng-Hua Bai, Shutian Liu, Shou Zhang, and Hong-Fu Wang. Photon blockade in a double-cavity optomechanical system with nonreciprocal coupling. New Journal of Physics, 22(9):093006, sep 2020 .

[33] Marc-Antoine Lemonde, Nicolas Didier, and Aashish A. Clerk. Antibunching and unconventional photon blockade with gaussian squeezed states. Phys. Rev. A, 90:063824, Dec 2014.

[34] M. Saffman, T. G. Walker, and K. Mølmer. Quantum information with rydberg atoms. Rev. Mod. Phys., 82:2313-2363, Aug 2010.

[35] M Saffman. Quantum computing with atomic qubits and rydberg interactions: progress and challenges. Journal of Physics B: Atomic, Molecular and Optical Physics, 49(20):202001, oct 2016.

[36] O Firstenberg, C S Adams, and S Hofferberth. Nonlinear quantum optics mediated by rydberg interactions. Journal of Physics B: Atomic, Molecular and Optical Physics, 49(15):152003, jun 2016.

[37] Jin-Feng Huang, Jie-Qiao Liao, and C. P. Sun. Photon blockade induced by atoms with rydberg coupling. Phys. Rev. A, 87:023822, Feb 2013.

[38] A Grankin, E Brion, E Bimbard, R Boddeda, I Usmani, A Ourjoumtsev, and P Grangier. Quantum statistics of light transmitted through an intracavity rydberg medium. New Journal of Physics, 16(4):043020, apr 2014 .

[39] J. D. Pritchard, C. S. Adams, and K. Mølmer. Correlated photon emission from multiatom rydberg dark states. Phys. Rev. Lett., 108:043601, Jan 2012.

[40] K. Hou, C. J. Zhu, Y. P. Yang, and G. S. Agarwal. Interfering pathways for photon blockade in cavity qed with one and two qubits. Phys. Rev. A, 100:063817, Dec 2019.

[41] C. J. Zhu, K. Hou, Y. P. Yang, and L. Deng. Hybrid level anharmonicity and interference-induced photon blockade in a two-qubit cavity qed system with dipole-dipole interaction. Photon. Res., 9(7):1264-1271, Jul 2021.

[42] Marc-Oliver Pleinert, Joachim von Zanthier, and Girish S. Agarwal. Hyperradiance from collective behavior of coherently driven atoms. Optica, 4(7):779-785, Jul 2017.

[43] C. J. Zhu, Y. P. Yang, and G. S. Agarwal. Collective multiphoton blockade in cavity quantum electrodynamics. Phys. Rev. A, 95:063842, Jun 2017. 
[44] Bastian Hacker, Stephan Welte, Severin Daiss, Armin Shaukat, Stephan Ritter, Lin Li, and Gerhard Rempe. Deterministic creation of entangled atom-light schrödinger-cat states. Nat. Photonics, 13:110-115, Jan 2019 .

[45] L. Béguin, A. Vernier, R. Chicireanu, T. Lahaye, and A. Browaeys. Direct measurement of the van der waals interaction between two rydberg atoms. Phys. Rev. Lett., 110:263201, Jun 2013.

[46] I. I. Beterov, I. I. Ryabtsev, D. B. Tretyakov, and V. M. Entin. Quasiclassical calculations of blackbodyradiation-induced depopulation rates and effective lifetimes of rydberg $n s, n p$, and $n d$ alkali-metal atoms with $n \leq 80$. Phys. Rev. A, 79:052504, May 2009 .

[47] Yu-Guo Liu, Keyu Xia, and Shi-Liang Zhu. Efficient microwave-to-optical single-photon conversion with a single flying circular rydberg atom. Opt. Express, 29(7):9942-9959, Mar 2021. 